ISSN: 2215-2644

revedu@gmail.com

Universidad de Costa Rica

Costa Rica

Percepciones sobre la lengua de señas chilena en la educación de estudiantes Sordos: docente y codocente como sujetos históricos situados comunicativamente en el aula ${ }^{[1]}$

\author{
Morales-Acosta, Gina \\ Percepciones sobre la lengua de señas chilena en la educación de estudiantes Sordos: docente y codocente \\ como sujetos históricos situados comunicativamente en el aula ${ }^{[1]}$ \\ Revista Educación, vol. 43, núm. 2, 2019 \\ Universidad de Costa Rica, Costa Rica \\ Disponible en: http://www.redalyc.org/articulo.oa?id=44058158007 \\ DOI: https://doi.org/10.15517/revedu.v43i2.31169
}

Esta obra está bajo una Licencia Creative Commons Atribución-NoComercial-SinDerivar 3.0 Internacional. 


\title{
Percepciones sobre la lengua de señas chilena en la educación de estudiantes Sordos: docente y codocente como sujetos históricos situados comunicativamente en el aula ${ }^{[1]}$
}

\author{
Perceptions of Chilean Sign for Teaching Deaf Students: Teaching and Co-teaching as Historical Subjects \\ Communicatively Positioned in the Classroom
}

Gina Morales-Acosta

DOI: https://doi.org/10.15517/revedu.v43i2.31169

Universidad Sek, Chile

Redalyc: http://www.redalyc.org/articulo.oa?id=44058158007

agafano@yahoo.com

Recepción: 04 Noviembre 2017

Aprobación: 29 Abril 2019

\section{RESUMEN:}

Este artículo parte de la tesis doctoral Sensibilidad intercultural: prácticas docentes con estudiantes Sordos de una escuela especial de Chile. Tiene como objetivo caracterizar las percepciones sobre la Lengua de Señas Chilena [LSCh] para la educación de los estudiantes sordo presentes en el personal directivo, docente y codocente del grado octavo. El estudio fue de tipo cualitativo, con un método de etnografía escolar con el estudio de caso de un aula de octavo grado de una escuela especial. La muestra fue un docente oyente, una codocente sorda y una directiva que daba clases o direccionaba las actividades educativas. Las técnicas usadas fueron entrevista en profundidad y observación con grabación audiovisual de siete clases con las correspondientes transcripciones, ortográfica de la entrevista y de glosa en lengua de señas. Se efectuó análisis de contenido con teoría fundamentada. Se muestra con el estudio que el docente y la co-docente de estudiantes Sordos tienen un posicionamiento comunicativo ético para el uso de la LSCh lo cual le ha permitido al estudiantado Sordo acceder a todas las áreas del conocimiento en el contexto escolar. Se enfrenta una diversidad de niveles de dominio de la LSCh en el aula de clase y la correspondiente diversidad en el desarrollo de la comprensión y expresión del lenguaje. Esto limita el desempeño del docente con respecto a lo que se está enseñando e impone roles adicionales que buscan compensar esas diversidades entre la escuela, la familia y otros contextos.

Palabras clave: Sujeto histórico, Comunicación, Lengua de señas, Sordo, Escuela Especial.

\section{Abstract:}

This article is based on the doctoral dissertation, Sensibilidad intercultural: prácticas docentes con estudiantes sordos de una escuela especial de Chile (Intercultural Sensitivity: Teaching Practices for Deaf Children at a Special Education School in Chile). The objective is to characterize perception regarding Chilean Sign Language [Lengua de señas chilena - LSCh] used by an administrative board and eighth grade teachers and co-teachers to educate deaf students. This was a qualitative study that used an ethnographic method with case studies from an eight grade classroom at a Special Education School. The sample included a hearing teacher and a deaf co-teacher as well as an administrative board that organized teaching activities. Among the techniques used were in depth interviews and observation including recordings of seven class sessions, transcriptions and descriptions in sign language. Content analysis was based on grounded theory. The study shows that teachers and coteachers of deaf students had better ethical communicational positioning regarding the use of sign language in order to provide students access to all areas of knowledge within the context of the school. The students in the class were not at the same level of mastery of LSCh, making it challenging for everyone in the classroom to understand and express themselves in sign language as well as limits the role of the teacher with regards to the material being taught. This requires additional roles within the school, family and other contexts to help compensate these differences.

KEYWORDS: Historical Subject, Communication, Sign Language, Deaf, Special Schools.

\section{INTRODUCCIÓN}

A lo largo de la historia, las personas Sordas ${ }^{[2]}$ han sido percibidas como sujetos con un déficit cognitivo o como incapaces al no comunicarse como la mayoría oyente, excluyéndolas de una participación social, económica, política, religiosa y educativa, negando derechos como el derecho al matrimonio, a heredar bienes 
y al negarles ser tratados lingüística y comunicativamente como personas menores que serán adultas y parte de una cultura Sorda que les es propia.

En relación con los derechos educativos en correlación con los derechos lingüísticos, actualmente, en Chile no se encuentran lineamientos en políticas lingüísticas los cuales propendan por la adquisición y desarrollo de la lengua de señas, como la lengua materna o primera lengua de una persona Sordo. Esto se evidencia al momento del ingreso a la escuela especial de estudiantado Sordo, a la cual llegan, en la mayoría de casos, sin conocimientos previos o con manejos básicos de la lengua de señas que les es propia, en este caso la Lengua de Señas Chilena (LSCh). La LSCh se reconoce como la lengua propia de los Sordos mediante la Ley N 20.422 del 2010 cuando se refiere en el Artículo 26 "la lengua de señas como medio de comunicación natural de la comunidad Sorda” (Ministerio de Planificación, 2010), otorgando el estatus lingüístico a la lengua implicada en diversos contextos sociales.

Aunque, cabe explicar que por sí sola, la ley es insuficiente para garantizar todos los derechos lingüísticos de las personas Sordas, pues se requiere un cuerpo normativo y de políticas lingüísticas que expliciten, en términos de las maneras y recursos, cómo será posible que la persona Sorda desde su nacimiento tenga garantía de acceso y desarrollo de la lengua de señas, así como su vivencia en los diferentes contextos como la familia, la escuela y el trabajo, principalmente.

\section{ANTECEDENTES}

Hablar de las personas Sordas sin decir su representación poblacional para Chile, es no dimensionar su magnitud e impacto. El estudio del Servicio Nacional de Discapacidad [SENADIS] expone un estimado del $12,7 \%$ de personas con discapacidad en el país, lo que supondría un aproximado en 2004 de 2,2 millones y un aumento a 2,6 millones de personas en situación de discapacidad con el estudio de 2015. De este conjunto para 2004 declararon que 488.511 personas tenían pérdida auditiva y 1.114 .911 de personas refirieron tener dificultades para oír en 2015, el equivalente al 6,9\% de la población total del país (SENADIS, 2006, 2015). Aunque es necesario decir que no se discriminan los datos según las causas de la Sordera que diferencian aspectos de la adquisición y desarrollo del lenguaje y su base en una lengua.

Dentro de los anteriores datos, se sabe que el $5 \%$ de la población Sorda tiene padres-madres Sordos (Schein, 1989 en Hadjikakou, Christodoulou, Hadjidemetri, Konidari y Nicolaou, 2009, p. 486) y por tanto tienen mayores posibilidades de acceso a una lengua de señas materna temprana; mientras que el restante $95 \%$ tienen padres oyentes, lo que da cabida a un abanico de posibilidades, bastante variable, que inicia con el momento del nacimiento cuando los padres y madres vivencian o no diferentes niveles de manejo de la lengua de señas para acompañar el desarrollo del lenguaje de su hijo o hija (Massone, Buscaglia y Cvejanov, 2012).

Aunque es complejo dar cuenta de datos relacionados con el número y formas de adquisición y desarrollo de la lengua de señas y del lenguaje en personas Sordas, como antecedente previo al ingreso a la escuela en Chile, es posible establecer datos relacionados como la escolaridad en esta población. De entrada, se reconoce que presentan proporciones de escolaridad desiguales al resto de la población. Así, se sabe que solo el 3\% de los niños, niñas y jóvenes Sordos en edad escolar van efectivamente a una institución educativa. Según datos, tomados del informe Antecedentes históricos: presente y futuro de la Educación Especial en Chile 2004, se identificó que la matrícula de educación especial en los años 1990-2001 para discapacidad auditiva, comprendió para el año 1990 un total de matrícula de 1.146 estudiantes y en 2001 un total de matrícula de 1.100 estudiantes (Godoy, Meza y Salazar, 2004). Este dato en relación con el estimado de la población Sorda en edad escolar, en general, estaría por debajo del porcentaje de niñas y niños oyentes matriculados en Chile al tomar como base que para 1991 la tasa de matrícula era de 95\% para escolares entre 6 a 13 años y de $77 \%$ para escolares entre 14 y 17 años.

Sí se ubica que para la población de niños y niñas hay políticas de la primera infancia en el programa Chile Crece Contigo, se cuestiona ¿qué medidas se estarían tomando para el ingreso escolar del $97 \%$ de niños, niñas 
y jóvenes Sordos que se encuentran fuera del proceso escolar?, ¿qué explica porcentajes de escolarización dispares para el estudiantado Sordo y el resto de la población? No es solo un hecho comparativo, pues el acceso a la educación se encuentra avalado por los Derechos de las niñas, niños y las personas en situación de discapacidad (ONU, 2006) y en lo establecido por Educación para Todos 2000-2015: Logros y desafíos (UNESCO, 2015) con lineamientos de inclusión en contextos educativos y lingüísticos propios, en consecuencia, se estaría hablando de niñas y niños Sordos amparados por los derechos humanos.

Por otro lado, ingresar a la educación debería considerar los derechos lingüísticos en esencia. El ser humano precisa una lengua, en el caso de las personas Sordas la lengua de señas, para las representaciones, espiritualidad y cosmovisión en lo más básico de la dignidad humana y que se entiende como "el derecho que cada comunidad lingüística tiene para dar forma a la vida en su lengua propia y en todos los ámbitos" (UNESCO, 1998, p. 9). El estado chileno no cuenta de forma clara con políticas lingüísticas en lengua de señas para involucrar a la familia o cuidadores, esto con el fin de propiciar estimulación lingüística temprana buscando que el entorno social esté constituido por interlocutores en lengua de señas (Ramírez y Masutti, 2009). Es decir, en generar un ambiente donde la palabra signada fluya en pleno derecho a crecer y tener la posibilidad de constituirse como sujeto en medio de una cultura oyente mayoritaria, sin estar como un manos atadas (Anzola, 1996).

Según lo anterior, se establecen las condiciones para que bebés Sordos puedan apropiarse de la lengua de señas como lengua materna que le posibilitará, en años posteriores, un mejor dominio de todos los niveles del lenguaje evitando que el niño o la niña hagan uso de códigos restringidos o caseros que, generalmente, solo son comprendidos por la familia o círculo social más inmediato, limitando de esa manera todas las posibilidades de participación y desarrollo dentro de una comunidad dada.

\section{MARCo TEÓRICo}

La educación de la persona Sorda, muestra el punto de enunciación comunicativa en el cual se han posicionado las directivas, docentes y codocentes como sujetos situados en el abordaje pedagógico (Zemelman, 2010). Así, un punto de enunciación sería el correspondiente a un modelo médico, el cual se centra únicamente en el método oralista puro, promoviendo prácticas logocéntricas. Los Sordos lo denominan el holocausto de la lengua (Ladd, 2011), que les privó de la comunicación en su lengua, la lengua de señas, por más de un siglo como forma básica de la dignidad humana (UNESCO, 1998). O el punto de enunciación del modelo social, en reivindicaciones o reconocimiento de la lengua de señas como una lengua y como propia del Sordo. Modelo social avalado con desarrollos e investigaciones lingüísticas propias de la lengua de señas de cada país. Algunos referentes de investigaciones de la lengua de señas en Latinoamérica son: el Primer diccionario bilingüe de Argentina escrito por Massone y Johnson (1993); Apuntes para la gramática de la lengua de señas colombiana de Oviedo (2001); El diccionario bilingüe de la lengua de señas chilenaespañol de Adamo, Acuña y Cabrera (2009) y el Diccionario español-lengua de señas mexicana de Acosta, Calvo, Maya y Sanabria, (2004). Además, Brasil cuenta con un centro de investigación en la Universidad de Santa Catarina dirigido por investigadores Sordos de nivel de doctorado que desarrollan pesquisas en análisis de su propia lengua.

El punto de enunciación, sirve a la vez como indicio de la búsqueda de un posicionamiento situado de acciones comunicativas para la educación de la persona Sorda. Así mismo, se reconocerá o no la lengua materna que les es propia a las personas Sordas, la lengua de señas (Bellés, 1995).

La educación especial, independiente del método de comunicación para Sordos establecido en el aula, también puede evidenciar "estereotipos que la sociedad les otorga a las personas con discapacidad auditiva a causa de la deficiencia, opacando sus competencias, en contextos cotidianos y enfocados a la hegemonía dominante la lengua oral" (Zondek, Zepeda, González y Recabarren, 2006, p.55). Aún más desalentador es el hecho de las bajas expectativas de éxito escolar para la comunidad Sorda, al respeto afirma Skliar (2003) que, 
Por lo general la educación de los Sordos, así como toda práctica que aborda las deficiencias, elude un debate educativo profundo y así induce inevitablemente a las bajas expectativas pedagógicas; los fracasos escolares, entonces, son fácilmente atribuidos a las supuestas culpas naturales de los propios deficientes (p. 2).

En este panorama, se identifica que hay vacíos en relación con cómo se entiende la educación de la persona Sorda y las maneras en que la misma tiene impactos positivos o negativos que no sean atribuibles a la deficiencia auditiva per se. Esto implica desconocer otras categorías y variables de análisis que influyen y determinan como es el caso del acceso, como derecho lingüístico, a la lengua de señas como lengua materna.

Enmarcar a las directivas, docentes y codocentes de estudiantes Sordos y Sordas como sujetos situados comunicativamente, implica poderlos nombrar también como sujetos históricos. En este sentido, el sujeto histórico es,

aquel capaz de ubicar al conocimiento que construye en tanto parte de sus opciones de vida y de sociedad. Esto implica tener que romper la tendencia a cosificar la realidad como simple externalidad, que envuelve a los sujetos de manera inexorable, para concebirla como una constelación de ámbitos de sentidos posibles (Zemelman, 2010, p. 2).

Según lo anterior, las directivas, docentes y codocentes tendrán que enfrentarse en su práctica a cambios de la transición comunicativa del estudiantado Sordo, es decir, nuevas formas para asumir la lengua de señas en el aula. Se asume, entonces, que cada actor educativo está situado en la acción educativa de la escuela como

Un ser con historia, es y está en y con el mundo por tanto es un sujeto social, cultural e histórico; este estar situado no significa determinación y acabamiento, sino por el contrario, posibilidad de ser: significa colocarse frente a la historia del hombre como la aventura de construirse como sujeto (Zemelman, 1998, p. 56).

Es decir, que las directivas, docentes y codocentes tienen tres componentes de decisión en relación con la situación comunicativa del contexto escolar y en particular del aula de clase, a saber: 1) cuentan con un proceso histórico comunicativo que da cuenta de las decisiones que tomaron en cada momento que los llevó a ser docente o codocente de personas Sordas; 2) han hecho una elección en relación con los métodos comunicativos para la educación del Sordo; y 3 ) tienen una postura o relación con la vivencia de la Sordedad o la cultura Sorda.

Este construirse como sujetos históricos para estar situados comunicativamente en el reconocimiento de la lengua de señas y considerar su uso, vivencia y desarrollo dentro del aula que les lleva a replantearse los métodos comunicativos utilizados en la educación de la persona Sorda que han sido impuestos desde la institucionalidad. En esta imposición, se consideran dos formas, por un lado, el uso de la lengua oral como punto y modo de articulación audio vocal, donde no todos los infantes Sordos logran acceder de forma efectiva a esta. Quienes no logran acceder requieren entrenamiento por personal especializado, terapias, y aditamentos auditivos como son audífonos o implantes cocleares. Por otro, el uso de un sistema mixto bimodal o comunicación total que es el uso simultáneo de algunos apuntadores de lengua de señas y palabras de forma oral.

Los métodos comunicativos en la educación de los Sordos, en el posicionamiento dialógico, enfrentan un cambio de paradigma relevante en la escuela (Marchesi, Alonso, Valmaseda y Paniagua, 1995). Cambio que pueden aludir a la transición en el papel o declarar abiertamente la aceptación e inclusión de la lengua de señas. Sin embargo, es necesario entender que dentro del aula docentes y codocentes son autónomos en sus prácticas, en ausencia de políticas lingüísticas y educativas para estudiantes Sordos que dispongan lo contrario, y por tanto pueden o no continuar privilegiando una lengua sobre otra. Es decir, continuar con trayectorias que privilegian la lengua oral a la lengua de señas. En una reflexión del estatus lingüístico de ambas, esto se daría por diferentes razones que pueden ser desconocimiento de la Sordedad y la cultura Sorda, falta de entrenamiento o interrelación comunicativa de forma simétrica con la comunidad y adultos Sordos que posicione el derecho a una lengua que le sea propia y por la cual se puedan expresar en lógicas y construcciones de identidad propias, como se los posibilita la lengua de señas. 
Cuando se privilegian, en diferentes niveles, los métodos comunicativos se promueven, a su vez, espacios en la escuela para las terapias orientadas a niñas y niños en el uso de un sistema lingüístico oral de forma temprana. Un resumen de los Métodos comunicativos más utilizados en la educación de las y los estudiantes Sordos que privilegian la lengua oral, o auditivo-vocal, se presenta en la Tabla 1.

TABLA 1

Métodos comunicativos utilizados en la educación de las y los estudiantes Sordos

\begin{tabular}{|c|c|c|}
\hline $\begin{array}{l}\text { Formato de la } \\
\text { lengua }\end{array}$ & $\begin{array}{l}\text { Estimulo } \\
\text { sensorial }\end{array}$ & Nombre de los métodos \\
\hline \multirow{8}{*}{$\begin{array}{l}\text { Lengua } \\
\text { auditivo-vocal } \\
\text { (Oral) }\end{array}$} & Unisensoriales & $\begin{array}{l}\text { Método Verbotonal } \\
\text { Terapia Auditiva Verbal }\end{array}$ \\
\hline & \multirow{3}{*}{ Multisensoriales } & $\begin{array}{l}\text { Sistema Fonológico de } \\
\text { Ling }\end{array}$ \\
\hline & & $\begin{array}{l}\text { Sistema Multisensorial } \\
\text { de Calvert }\end{array}$ \\
\hline & & $\begin{array}{l}\text { Método reflexivo de Van } \\
\text { Uden }\end{array}$ \\
\hline & \multirow{3}{*}{$\begin{array}{l}\text { Audio vocales } \\
\text { basados en la } \\
\text { lectura labial }\end{array}$} & $\begin{array}{l}\text { El abecedario silábico de } \\
\text { Hongtai }\end{array}$ \\
\hline & & $\begin{array}{l}\text { El alfabeto de Kinemas } \\
\text { asistidos (AKA) }\end{array}$ \\
\hline & & $\begin{array}{l}\text { La Palabra } \\
\text { Complementada (Cued } \\
\text { Speech) }\end{array}$ \\
\hline & Mixtos & $\begin{array}{l}\text { Método bimodal - Cued } \\
\text { speech o la palabra } \\
\text { complementada (LPC). }\end{array}$ \\
\hline
\end{tabular}

Fuente: Adaptado de Mantas-Cárdenas, R. (2014, p. 12).

Por su parte, la lengua de señas tiene como características propias tener una estructura visogestual y, el uso del cuerpo y las manos en la adquisición y desarrollo. Además, la lengua de señas hace uso de un sistema dactilológico en el deletreo manual (configuración manual del abecedario), que apoya la adquisición de la lengua escrita. La escritura en las y los Sordos es tomada como adquisición de una segunda lengua (L2) con apoyo visual de la primera, que es la lengua de señas como (L1).

Se observa, que históricamente, la lengua oral ha sido eje central de los métodos comunicativos utilizados en la educación de Sordos. Aunque, se reconoce que las personas Sordas, entre las formas de comunicación, prefieren el uso de la lengua de señas, bien la pueden utilizar de forma principal o alterna a la oral o escrita. En esta línea de ideas, se encuentran códigos limitados como sistemas de gestos manuales o caseros, que son propios de las personas que no acceden al contacto directo con la comunidad Sorda en apropiación de una cultura, adquisición y desarrollo de una lengua a edades tempranas, dejándolos al margen de una pertenencia e identidad cultural (Carvajal, Tascón y Vásquez, 2012).

Por lo anterior, es difícil determinar, en las interacciones cotidianas, una exclusiva forma de comunicación. Por ende, cabría afirmar que en las diversas formas que utilizan las personas Sordas existe preferencia por una forma u otra de comunicación según las demandas del contexto y la actividad que desempeñan. Estas situaciones comunicativas que emergen de las interacciones, entre Sordos y entre Sorda(o)-oyentes, conllevan a la escuela a tener que reflexionar críticamente sobre los métodos comunicativos utilizados en la educación de la persona Sorda, para tomar una posición lingüística clara en relación con la lengua de señas que permita desarrollos académicos y curriculares, sobre todo acordes al desarrollo y el respeto de la identidad de los estudiantes Sordos.

$\mathrm{Al}$ referir situaciones comunicativas de paso del hogar al contexto escolar convoca a que las y los docentes y codocentes, al ser sujetos históricos situados comunicativamente, encaren la tarea de tomar una posición 
lingüística decisoria en relación con la LSCh para la educación de Sordos, a expensas de no tener y buscar ser apoyados, en el futuro, por políticas lingüísticas y educativas precisas sobre el tema. Así, la escuela requiere adecuar el entorno para propiciar el contacto con docentes bilingües o modelos lingüísticos Sordos que favorezcan la adquisición de la lengua, el desarrollo de la lectura y la escritura como segunda lengua en los Sordos (Pinto, 2012). Adecuar, desde el punto de vista pedagógico, se entendería como el establecimiento general de las condiciones para que se dé un bilingüismo construido. El bilingüismo no se adquiere de manera espontánea, requiere estar expuesto en forma activa a la lengua materna del Sordo, la lengua de señas, y explicar-enseñar la segunda lengua, la lengua escrita, con adecuaciones visuales pertinentes a estudiantes Sordos.

El panorama para desarrollar el bilingüismo parte de la primera lengua, que en muchas situaciones comunicativas describe a hijas e hijos Sordos con padres oyentes que llegan a la escuela con un repertorio comunicativo limitado. Es indispensable que existan unas condiciones adecuadas en la escuela para que el niño y la niña Sorda potencien su primera lengua (L1). Un paso inicial e importante para comprender el bilingüismo en Sordos es poder verlos como usuarios de la lengua de señas como su primera lengua y que tiene formato visogestual. A la vez, que la segunda lengua de su bilingüismo es la Lengua escrita (L2).

Estas condiciones de bilingüismo construido, en el marco de los lineamientos de Educación para Todos y todas, han sido asumidas de diferentes maneras por los gobiernos de Camerún, Colombia, Ghana y Kenia. Incluido el tema de la educación de los Sordos con la adquisición de la primera lengua como parte de sus respectivos lineamientos de primera infancia. Sin embargo, según el informe del Banco Mundial (2013) el programa de educación aumentó en Chile los recursos para niñas y niños de 4 y 5 años matriculados en educación preescolar en general y no refiere datos o inclusión de recursos para estudiantes Sordos.

Asimismo, en el marco de la apuesta del Gobierno de Chile en Educación para Todos 2000-2015: Logros y desafíos (Unesco, 2015) se proporcionan textos escolares obligatorios de manera gratuita para todos los grados académicos, algunos incluyen adecuaciones para discapacidad sensorial en accesibilidad a la lectura en Braille y un tipo de letra más grande o macrotipo para estudiantes de baja visión, sin embargo, en el conjunto de dichos textos escolares no se encuentran adaptaciones para estudiantes Sordos.

De la misma manera, se promueve la asignación a escuelas públicas de enseñanza básica y media recursos para la tecnología a través de software, conexión y apoyo técnico mediante el programa Enlaces con TICS, no obstante, se observa en ocasiones un engranaje poco adecuado de la tecnología para estudiantes Sordos en el currículo o didáctica para el aprendizaje de la primera y segunda lengua en Sordos (Salinas, Sánchez, Purcell y Mendoza, 2009). Es decir, usar la tecnología de forma aislada sin estar en mediación con la lengua de señas, no construye bilingüismo, ni guía el aprendizaje de la primera lengua (señas) a la segunda lengua (escritura). Pensar entonces una educación bilingüe para estudiantado Sordo, que incluya a todas las personas desde espacios tecnológicos en el aula de clase debe abordar la lengua de señas con aspectos básicos de estrategias visuales y kinestésicas que propicien la lengua como tal en el aula (Ramírez y Masutti, 2009).

Lo anterior, desde un enfoque pedagógico en el cual se concibe la competencia comunicativa delimitada como "La capacidad del maestro para establecer una comunicación efectiva y eficientemente óptima [con sus estudiantes], al desarrollar en su personalidad un estilo de comunicación flexible y lograr resultados educativos deseados" (Ortiz, 1997 citado en Aguirre, 2005, p.5). Así, la concepción del docente o la codocente como sujetos históricos situados comunicativamente tiene todo el sentido para la comprensión del establecimiento de una comunicación flexible y efectiva para la enseñanza y el aporte al desarrollo del bilingüismo en los y las estudiantes Sordos, cuando el situarse comunicativamente se hace en la lengua de señas.

A partir de lo argumentado hasta el momento, se plantea en la escuela especial la consideración para el posicionamiento comunicativo con la lengua de señas: el acceso temprano y la fluidez de la lengua de señas chilena de las y los estudiantes Sordos y las correspondientes percepciones de los y las docentes, las y los codocentes y directivas sobre estos aspectos que influyen sobre sus prácticas. Sin embargo, las percepciones y 
su respectiva incidencia en las prácticas dependerán de la manera en que docentes y codocentes se posicionen como sujetos históricos situados comunicativamente en el aula y que abogará, por un lado, a usar métodos comunicativos (oralidad o bimodales) o, por el otro, la lengua de señas chilena. Se respalda que al considerar los principios interculturales como aquellos que promueven adecuaciones según las formas de construcción de identidad entre culturas, se haga uso de elementos visuales para el currículo y una metodología propia para la cultura Sorda en el contexto escolar y en el aula, y, por tanto, del bilingüismo en el docente y el uso de la lengua de señas chilena para la enseñanza.

El acceso temprano a la lengua de señas es también la posibilidad de acceso temprano a la construcción de identidad en Sordedad (Ladd, 2011) y cultura Sorda, que lleva a inquirir por las circunstancias que llevaron a la elección y uso de métodos comunicativos en la educación del estudiantado Sordo hacia la transición, explícita, del uso de la lengua de señas chilena para aspectos pedagógicos en contextos propios. Al trabajar en el aula docentes y codocentes tendrán acceso la a diversidad Sorda en diferentes niveles y maneras de comunicación: estudiantes que hablan, que hacen señas, que realizan bimodal, como también diversas formas de acceso al sistema educativo e ingreso extra edad, para procesos de enseñanza aprendizaje. Bajo este panorama ¿cuál es la percepción sobre la lengua de señas chilena para la educación del estudiantado Sordo presente en el personal directivo, docente y codocente como sujeto histórico situado comunicativamente en la escuela especial en uso y reconocimiento de la lengua de señas chilena?

\section{MÉTODo}

El estudio tuvo un enfoque cualitativo, orientada bajo la referencia epistemológica del paradigma interpretativo y diseño de la etnografía escolar con la selección de un caso para el escenario de investigación, que fue una escuela especial para el estudiantado Sordo. Se tuvo como orientación para la investigación cualitativa a Zemelman (2010), en cuanto a la importancia vital que el sujeto investigado se posicione comunicativamente como sujeto histórico de sus saberes y que de forma crítica reflexiona sobre las razones de una realidad social de la que es parte.

La selección de la escuela especial para personas Sordas de la región Metropolitana de Chile, de las cuatro que existen en esta región, se hizo teniendo en cuenta que una de ellas en los últimos ocho años tuvo acciones de transición de los métodos comunicativos utilizados en la educación de las personas Sordas al uso de la LSCh. La escuela especial es una institución de la Municipalidad Santiago Centro, pública, con carácter de colegio municipal que acoge a niños, niñas y jóvenes en los niveles: Maternal, Pre-Básico y Básico en la modalidad de enseñanza diurna con dos jornadas, una de solo en la mañana y otra de jornada escolar completa diurna. La escuela cuenta con 26 profesores y profesoras; 26 cursos distribuidos en nueve de prebásica y 17 de básica; una matrícula aproximada de 134 estudiantes en edades que fluctúan entre los cuatro y diecisiete años, provenientes de todas las comunas de la región Metropolitana.

Los criterios aplicados a la escuela especial, como unidad de investigación, para seleccionar a la población muestra de docentes y codocentes fueron: que manejaran de forma única o alterna la LSCh (bilingües); que conocieran los métodos comunicativos utilizados en la educación de la comunidad Sorda como son oralización o bimodal; que reconocieran la escritura como segunda lengua en la comunidad Sorda o bilingüismo (L2); que las exigencias comunicativas en lengua de señas de los cursos en los que estuvieran fueran mayores; que tuvieran mayor tiempo de servicio con la población Sorda en la escuela especial; mayor nivel de experiencia en educación con personas Sordas en general; alto nivel de receptividad hacia la cultura Sorda; y que, por supuesto, estuvieran de acuerdo con participar en la investigación. Además, reconociendo que en Chile las y los estudiantes Sordos asisten a la escuela de básica primaria hasta octavo grado y de ahí egresan a un Programa de Integración Escolar (PIE) ${ }^{[3]}$ siendo este el nivel de mayor exigencia en el uso de la LSCh de la escuela especial. Entonces se seleccionó como muestra un docente y una codocente Sorda con segmentos de clase para el grupo de octavo grado, grupo de ocho estudiantes jóvenes en dominio de diferentes 
niveles de la lengua de señas y los métodos de comunicación. La muestra se completó con la directiva de la escuela especial que es quien ha estado al frente de la transición al uso de la LSCh.

Como herramienta para la recolección de datos se utilizó la técnica de entrevista en profundidad que se aplicó a la directora, un docente y una codocente Sorda de la institución. Las entrevistas se hicieron de forma individual con registro de grabación audiovisual y notas de contexto de la investigadora, así como intérprete en LSCh para acompañar la entrevista a la codocente Sorda. Como pautas para las entrevistas a profundidad se tuvo el derrotero descrito en la Tabla 2: Pautas de entrevista a profundidad según categorías de la investigación.

TABLA 2

Pautas de entrevista a profundidad según las categorías de investigación

\begin{tabular}{|c|c|c|}
\hline $\begin{array}{l}\text { Pregunta } \\
\text { Investigativa }\end{array}$ & Objetivo & Categorias \\
\hline \multirow{10}{*}{$\begin{array}{l}\text { ¿Cuál es la } \\
\text { percepción sobre la } \\
\text { LSCh para la } \\
\text { educación el } \\
\text { estudiante Sordo } \\
\text { presente en el } \\
\text { personal directivo, } \\
\text { docente y codocente } \\
\text { como sujeto } \\
\text { histórico situado } \\
\text { comunicativamente } \\
\text { en la escuela } \\
\text { especial? }\end{array}$} & \multirow{10}{*}{$\begin{array}{l}\text { Caracterizar la } \\
\text { percepción sobre la } \\
\text { LSCh para la } \\
\text { educación el } \\
\text { estudiante Sordo } \\
\text { presente en el } \\
\text { personal directivo, } \\
\text { docente y } \\
\text { codocente como } \\
\text { sujeto histórico } \\
\text { situado } \\
\text { comunicativamente } \\
\text { en la escuela } \\
\text { especial. }\end{array}$} & Sujeto Histórico Situado \\
\hline & & $\begin{array}{l}\text { ¿Cómo es su historia para } \\
\text { Ser docente de Sordos? }\end{array}$ \\
\hline & & $\begin{array}{l}\text { ¿Cuántos años lleva como } \\
\text { docente en la escuela? }\end{array}$ \\
\hline & & $\begin{array}{l}\text { ¿Cómo ha sido la } \\
\text { experiencia de enseñar en } \\
\text { el colegio? }\end{array}$ \\
\hline & & $\begin{array}{l}\text { ¿Cómo es su relación con la } \\
\text { comunidad Sorda en el } \\
\text { colegio y la asociación? }\end{array}$ \\
\hline & & Sordedad \\
\hline & & $\begin{array}{l}\text { ¿Asiste a la asociación o } \\
\text { comparte en otros espacios } \\
\text { con Sordos adultos fuera } \\
\text { de la escuela? }\end{array}$ \\
\hline & & $\begin{array}{l}\text { ¿Cómo integrar este } \\
\text { conocimiento con los } \\
\text { estudiantes con diversidad } \\
\text { Sorda en el aula? }\end{array}$ \\
\hline & & Métodos comunicativos \\
\hline & & $\begin{array}{l}\text { ¿De qué forma o formas se } \\
\text { comunica con sus } \\
\text { estudiantes Sordos en el } \\
\text { aula de clase? }\end{array}$ \\
\hline
\end{tabular}

Fuente: Morales-Acosta, G. (2016).

Las entrevistas a profundidad fueron transcritas al español escrito en el caso de los registros en audio. Para los datos visuales en LSCh se hizo transcripción en glosa al español escrito con apoyo de un intérprete en LSCh para esta actividad.

El análisis fue de contenido y se hizo con fundamentación en la teoría y siguiendo lo propuesto por Berelson, para lo cual, se pasó a ejecutar el proceso de análisis con la preparación de datos a partir de las transcripciones escritas y en glosa para la construcción de la unidad de análisis y la correspondiente organización con la selección de codificación y selección de citas (Berelson, 1952). Con lo anterior fue posible la creación de relaciones entre categorías y elementos de categorías para dar sentido a las percepciones de la directiva, el docente y la codocente sobre la mirada que tienen de la LSCh para la educación del y la estudiante Sordo. Una vez establecidas las relaciones se pudo llegar a la descripción de resultados según categorías de análisis. Durante todo el proceso se hizo búsqueda y selección de datos significativos en los corpus que componían la unidad de análisis, se documentó con la opción de notas de la investigadora y se generó el informe. 
Los códigos asignados fueron: para el docente de D01, para la codocente Sorda de D02 y para la directora de D03. Un ejemplo de la segmentación y codificación del conjunto de datos de las entrevistas se presenta más adelante, donde la letra $\mathrm{P}$ hace referencia a el párrafo y está seguida del número del mismo en el corpus de las tres entrevistas. Así, este ejemplo, agrupa citas relacionadas con una misma categoría (ver Tabla 3).

TABLA 3

Ejemplo de segmentación y codificación de datos

\begin{tabular}{|c|c|c|c|c|}
\hline \multirow[t]{2}{*}{ Segmento } & \multicolumn{4}{|c|}{ Codificación } \\
\hline & $\begin{array}{l}\text { No. } \\
\text { párrafo }\end{array}$ & Personal & $\operatorname{cod}$ & $\begin{array}{l}\text { Línea del } \\
\text { párrafo }\end{array}$ \\
\hline $\begin{array}{l}\text { P3: Entrevista } \\
\text { D01 (29). }\end{array}$ & 3 & Profesora & D01 & 29 \\
\hline $\begin{array}{l}\text { P4: Entrevista } \\
\text { D02 (118). }\end{array}$ & 4 & Codocente & $\mathrm{DO2}$ & 118 \\
\hline $\begin{array}{l}\text { P2: Entrevista } \\
\text { D03 (72). }\end{array}$ & 2 & Directora & $\mathrm{DO3}$ & 72 \\
\hline
\end{tabular}

Fuente: Morales, G. (2016).

\section{Resultados}

La descripción de los resultados muestra la mirada sobre la lengua de señas chilena presente en la directiva, el docente y la codocente como sujetos históricos situados comunicativamente en la escuela especial a partir de su proceso histórico comunicativo; los métodos comunicativos utilizados en la educación del y la estudiante Sordo, ya sean el Oral, Bimodal y la lengua de señas; y su relación con la Sordedad o la cultura Sorda.

\section{Proceso histórico comunicativo}

En historia comunicativa primero se presentará a la directiva en la historia de la institución y la toma de decisiones y en segundo lugar al docente y la codocente. La entrevista en profundidad destacó la historia de la institución fundada en 1960, la cual ha presenciado y contribuido a la educación de personas Sordas, por un lado, desde su génesis se concibió como oralista con una mirada clínica.

Hubo un tiempo en que entraban acá solo niños Sordos que tenían posibilidad de desarrollar lenguaje oral, todos los niños Sordos que tenían otras dificultades no entraban a esta escuela y así la escuela evolucionó año por año (P2: Entrevista D03 (41).

En segunda instancia, en el devenir histórico del país y del personal, la toma de decisiones como escuela especial y el replantear formas Otras, hacen que el establecimiento lleve aproximadamente nueve años de transición de los métodos de comunicación usados en la educación del y la estudiante Sordo en línea oralista y bimodal a el uso de la lengua de señas. Transición posible por un autoconstruir a través de prácticas e investigaciones para legitimar y activar el uso de la LSCh en la institución y dentro del aula.

Llegó un director que dijo iya! vamos a ser bilingües, vamos a ser un proyecto bilingüe (P2: Entrevista D03 (41).

Se devela, a su vez, que la anterior situación los llevó a posicionarse como sujetos históricos situados al tomar decisiones para considerar los procesos pedagógicos de inclusión para el futuro comunicativo de las y los estudiantes Sordos, aunque se encuentra limitaciones de normatividad y políticas lingüísticas, ante lo cual se encuentra que, 
Nuestro pilar la lengua de señas, pero por la ley de inclusión nuestros niños Sordos se están yendo a las escuelas regulares y en las escuelas regulares nadie sabe lo que es pedagogía para personas Sordas (P2: Entrevista D03- (148).

Nosotros queremos hacer cambio por el futuro de los chicos Sordos y por lo que ellos vivieron también eso ha da dado una relación muy de cariño y de respeto (P 2: Entrevista D03 (76).

Como antecedente de la historia de la educación superior recibida, se evidencia que no les entregó herramientas necesarias en comunicación en lengua de señas, aunque sí recibieron entrenamiento para los métodos de comunicación usados en la educación del y la estudiante Sordo en la línea oralista y bimodal.

Nunca hice mi practica en lenguaje y en ese tiempo los Sordos llamábamos multidéficit, salí de la Universidad en ese tiempo me fui a trabajar a una escuela de Sordos (P 2: Entrevista D03 (12).

Sin embargo, se reconoce que el cuerpo docente recién titulado egresa con conocimientos lingüísticos, didácticos y curriculares básicos, siendo en la práctica, donde adquiere bases de pedagogía del estudiantado Sordo en uso de la LSCh, por ende, logran desarrollar el vocabulario pedagógico pertinente al grado o área, donde le asignen impartir clase.

En relación con las percepciones sobre ¿cómo ha sido la experiencia de enseñar en el colegio? las respuestas indican un marco de inclusión, cercanía e intimidad por agradecimiento al referirse a la experiencia como hacer parte de una familia.

Aquí en la comunidad de Sordos me siento como una parte más de la familia, me siento como una tercera familia, hay mucha confianza (P4: Entrevista -D02 (28).

La directiva, el docente y la codocente reconocen y dan cuenta de la diversidad que se encuentra dentro de la institución. En este sentido, se identifica que se cuenta con docentes que tienen experiencia en la educación del estudiantado Sordo, las cuales oscilan entre 2 y 40 años. De estos las personas que integran este estudio tienen 17, 9 y 2 años de experiencia. Algunos(as) de ellos(as) tuvieron en su formación presencia de elementos de énfasis oralista y bimodal, y otros(as) llegaron con niveles diferentes de dominio de la LSCh, en que logran dar una clase de forma fluida o con apoyo de la codocente.

En la transición de la institución, también se reconoce que, si bien la misma por muchos años contó en su nómina con personas Sordas trabajando, éstas estaban en labores de vigilancia y aseo. Así la directiva, en coherencia con los lineamientos de uso y vivencia de la lengua de forma simétrica con visibilidad de las personas Sordas, efectúa contratación de docentes y codocentes Sordos. Estos docentes y codocentes pueden tener o no un nivel fluido en lengua de señas, o formación en énfasis oralista, ya que algunos son egresados del mismo colegio y vivenciaron la comunicación del modelo oral y bimodal como estudiantes, ahora como compañeros de trabajo, vivencian la transición a la lengua de señas en simetría comunicativa en relación con el personal directivo y docente oyentes para que estos conjuguen la experiencia anterior, ahora como personas adultas Sordas, con o sin procesos académicos para el aula.

Muchos Sordos codocentes de esta escuela estudiaron acá (P2: Entrevista D03 (48).

La relación de docentes con la comunidad Sorda en el colegio es desde el respeto y de docentes en construcción de autonomía y para resarcir antiguas prácticas en la institución con personas que en un momento histórico dado no respetaron su lengua.

La historia que tiene [...] (el o la) codocente es súper fuerte, súper marcada por una experiencia de vida, es súper complicado que ella se ponga al nivel de la profe o no quiere o simplemente la ve como una persona que nunca respetó su lengua (P2: Entrevista D03 (58).

Una codocente le tocó ir a una sala, porque antes cuando faltaba una profesora los cursos se dividían a esta persona, lo pasó pésimo siendo alumna (P 2: Entrevista D03 (56) 
Se refieren diferentes niveles de relación con la Sordedad en cuanto a las interacciones y reconocimiento de las asociaciones de y para personas Sordas, ya sea en la niñez o como adultos, y lo que esto implica en la práctica de una cultura Sorda.

Cuando era chica no sabía de la asociación de Sordos de la fuerza... (adulta) A la asociación antes iba al fútbol, iba a ver como jugaban los hombres, ahora en la asociación menos, porque está más lejos (P4: Entrevista - D02 (32).

Ahora no voy a la asociación cuando era estudiante sí... A muchos de los Sordos de acá los conocía antes en el club universo, deportivo está en avenida Matta, otros del Club real y la Asoch porque estaba cerca de la Universidad (P3: Entrevista D01 (42).

Estas participaciones muestran la asistencia restringida a solo eventos deportivos como debilidad en la existencia de espacios comunitarios comunicativos que permitan interactuar con otros hablantes nativos con fluidez en LSCh.

\section{Métodos comunicativos usados para la educación del estudiantado Sordo}

El método comunicativo utilizado en la educación de personas Sordas como la expresión del lenguaje humano, usado en los últimos cien años, ha sido con prelación un sistema verbal (Marchesi et ál., 1995), es decir, que una lengua tendría que considerar como componente verbal la lengua de señas y la lengua oral. Sin embargo, fue la segunda la que se instauró de dos formas en la escuela, una lengua oral y un sistema mixto (bimodal o comunicación total). El sistema bimodal se percibe, por la codocente Sorda, como una interrupción de la fluidez en el uso de las señas.

Pero los profesores eran oralistas, no había identidad (P4: Entrevista - D02 (32).

Yo crecí oralista, mi experiencia los profesores me enseñaron más oralista, cuando era el trabajo oralista, cuando eran clases en lengua de señas eran muy básicas eran señas como 'la casa' (P 4: Entrevista - D02 (67).

Me gusta más la lengua de señas, natural, que el bimodal, se siente que va interrumpiendo la seña (cuando hablan) parecido al español, la lengua de señas me gusta si (P4: Entrevista D02 (78).

El sujeto histórico situado comunicativamente en la acción educativa de la institución es el que logra tomar decisiones en reconocimiento y legitimación de la LSCh, como la lengua que le es propia a la persona Sorda y por tanto la que le da la posibilidad de construirse como sujeto. En este sentido, retomando Zemelman, se está ante lo que la LSCh aporta y es lo que "significa colocarse frente a la historia del hombre como la aventura de construirse como sujeto" (Zemelman, 1998, p. 56), teniendo como base una lengua para poder hacerlo. Este construirse para estar situado considerara el bilingüismo para personas Sordas, en cuanto que la Lengua de señas (L1) es la primera lengua y la Lengua escrita (L2) es la segunda lengua. En el marco de la escuela especial la transición al bilingüismo para poder ser sujeto situado comunicativamente como Sordo o Sorda es percibido como una lucha con abanderados y detractores.

Hay un grupo importante de profesoras bilingües que dieron la pelea durante años, gracias a ellas se formó un curso de bilingües que en verdad los niños con más dificultades son los que se tiraron a ese curso, ellas se la jugaron por eso, a pesar de eso, fueron súper maltratadas (P2: Entrevista D03 (218).

Las profesoras, abanderadas, conformaron un hito en la institución para el cambio del paradigma comunicativo de Oral-Bimodal a el bilingüismo de las personas Sordas en la práctica docente, ya no de manera individual, de minoría o resistencia, sino con acogida y puesta en marcha en la escuela al estar incluido en el Proyecto Educativo Institucional PEI.

Estos dos años hemos estado como haciendo ese proceso de sensibilizar que los profesores entiendan que es bilingüismo, que no es estar en la sala hablando y haciendo unas pocas señas para que el niño entienda (P2: Entrevista D02 (41). 
Bilingüismo es entregar todo el contenido de una clase en lengua de señas para que el niño efectivamente lo pueda interiorizar y luego ir dando de acuerdo a las características de cada niño estrategias que le sirvan a ellos (P 2: Entrevista D02 (41).

Cuando tú eres bilingüe intercultural tu pedagogía va a estar basada en estrategias visuales que tu lengua de señas sea cada vez más rica para que puedas entregar mejor contenido a los niños. En cambio, cuando estas parado en la pedagogía auditivo oral tu pedagogía va a ser oral y probablemente van a ser otras tus estrategias y tus objetivos entonces depende también de lo que tú quieras lograr (P2: Entrevista D03 - 2 (148:149).

Al considerar a la institución y al docente en un devenir histórico oralista con los métodos comunicativos para la educación del y la estudiante Sordo y el en contacto de una mirada académica Otra, se encuentra en la formación superior el reconocimiento de posibilidades liberadoras a la vez que poco desarrolladas para la transición a el bilingüismo.

Tengo un postítulo en psicopedagogía y ahora estoy haciendo otro magíster, pero, o sea, mientras uno es profesor tiene que estudiar porque esta cuestión es súper rápida (P2: Entrevista D03 (28).

Universidad me habían dado comunicación total, eso es lo que había en ese entonces y yo sabía que la cosa por el oralismo no iba (P2: Entrevista D02 (15).

Por lo anterior, la formación superior, guío los lineamientos que permitieron a los y las docentes, así como a la directiva, para posicionarse como sujetos e institución en la transición de una mirada puesta en un modelo clínico con soporte en lo oral a un modelo social con respeto a la lengua de señas.

\section{Sordedad}

La vivencia de la Sordedad, implica diversas dimensiones de lo cotidiano y como se organiza de lo auditivo a lo visual. En este sentido, los arreglos para cambiar el timbre sonoro por un timbre de luz móvil o baliza, como la que utilizan los carros de bomberos o ambulancias que poseen una luz giratoria, es legitimado y avalado por los y las Sordos de la escuela, e implica que otras poblaciones como los niños y niñas de retos múltiples reconocieran estos arreglos como una forma de comunicación visual posible para los manejos de los tiempos académicos y evacuación en situaciones de emergencia.

Qué sentido tiene para la comunidad Sorda y niños Sordos, cual es el sentido de tocar la campana, mirémoslo con otro sentido, son personas Sordas la visión es importante (P2: Entrevista D03- (106).

En el rescate de acciones a tener en cuenta como procesos de identidad Sorda, y un aporte para que las y los estudiantes Sordos reconozcan espacios, es la asociación como un pilar importante en la comunidad que le es propia. Además, se plantea la necesidad de contacto con la asociación en edades tempranas para comunicar y construir en identidad Sorda, en Sordedad.

Antes yo no sabía cuándo era chica no sabía de la asociación de Sordos de la fuerza (P4: Entrevista - D02- (32).

En la casa yo veía la tele y no hacía nada más, después con el tiempo vi los Sordos que hacía lengua de señas y me gustaba

(P4: Entrevista D02 (32).

Mi experiencia en la asociación, los encuentros Sordos, a veces hacemos encuentros para futbol, cuento más de la cultura, de fiestas, eventos, antes había más información (P4: Entrevista D02 (57).

Cuando entre a la asociación me gusto la socialización la comunicación, compartir, conversar (P4: Entrevista D02 (34). Sentí que mi identidad estaba en lengua de señas (P4: Entrevista D02 (34).

En la participación, del docente y la codocente Sordos con las y los estudiantes Sordos, para explicar el vivenciar desde su experiencia de o en la comunidad Sorda que les es propia se hace con elementos de contexto, estos les permiten poner en concreto lo que implica poder tener pares comunicativos en lengua de señas para la realización de actividades cotidianas, pues de otra manera se vivenciarían estas cotidianidades como aislamiento comunicativo. 
Sobre el tema de la comunidad Sorda, yo les explico, por ejemplo, hacen un campamento Sordo, yo nunca tuve esa experiencia no puedo mentirles, pero si contarles la importancia del campamento, la importancia de la asociación (P4: Entrevista D02 (52).

Ahora acá en la escuela se han integrado en la escuela muchos profesores (Sordos), allá afuera es otra situación (P4: Entrevista D02 (28).

Las relaciones entre docentes y codocentes instalan en la escuela formas de cultura oyente y cultura Sorda o en Sordedad al compartir espacios, aunque con dejes de resistencias históricas por predominio de la oralidad.

Tienen que aceptar, los profesores oyentes tienen que aceptar la cultura Sorda, abrir un poco la mente, que es parte de la cultura de los Sordos las luces que se toquen (llamando la atención en señas en el cuerpo) los Sordos llaman la atención pisando fuerte el suelo y dicen No, los oyentes dicen pisar así es feo es una falta de respeto y no, es falta de abrir la mente a la cultura Sorda (P4: Entrevista D02 (100).

Falta más enseñarles, eso de mirar es de la cultura Sorda, el encuentro visual (P4: Entrevista D02 (108).

Si no me mira [estudiante o colega] cómo voy a comunicarme, ahí se explica y toma conciencia (P4: Entrevista D02 (109).

Me preguntaron ¿qué significa rol? Que significa esa seña, significa que yo soy modelo, personaje le explique (P4: Entrevista D02 (128).

Para finalizar, estas descripciones de resultados, producto del análisis del discurso del personal directivo, docente y codocente como sujetos históricos situados para el uso de métodos comunicativos en punto de enunciación de la lengua de señas como lengua materna de la persona Sorda, se identifica que guiaron, y pueden dar luces para guiar acciones en el futuro, la revisión y puesta en práctica de iniciativas visuales pertinentes a los estudiantes Sordos para su educación en la escuela especial.

\section{Discusión}

Considerando los sentidos posibles y resignificaciones comunicativas de la directiva, el docente y la codocente para el uso de la lengua oral y la transición hacia la lengua de señas para las y los estudiantes Sordos en procesos de aprendizaje exigió, y sigue exigiendo de la institución, en primer lugar, el afianzar la lengua de señas de forma transversal y reconocer la diversidad Sorda de los estudiantes.

En segundo lugar, le demanda a docentes y codocentes tener acercamiento y conocimiento de la comunidad Sorda de una manera simétrica, que permita consultar a hablantes nativos adultos y desarrollar de manera conjunta el vocabulario pedagógico; a la vez que, del y la docente oyente bilingüe convivir en las prácticas educativas con colegas que dan prelación a la lengua oral, y que generalmente ingresan al salón para brindar información de forma oral, situación que excluye al codocente y estudiantes Sordos. Para esto último, requiere un ejercicio de respeto a los colegas como un sujeto histórico situado comunicativamente en polo opuesto comunicativo de ellos, en áreas del conocimiento en que no se utiliza plenamente un discurso en señas, sino órdenes simples, acciones, señalar o indicar, sin secuencia en coherencia y cohesión comunicativa.

Docentes y codocentes, al ser sujetos históricos situados comunicativamente, captan todo el tiempo señales comunicativas del estudiantado en diversidad Sorda y pueden optar por centrarse en el aula por abordar la emoción ante situaciones externas del colegio que afectan. $\mathrm{O}$ por el contrario omitirlas y dar prioridad al contenido de la clase y fomentar la intención comunicativa en el aula. También, deben analizar el nivel de lengua de señas que traen los estudiantes, y monitorear mediante su desarrollo y el grado o nivel en que se encuentran en el uso de la LSCh.

El o la docente como sujeto histórico comunicativo situado se asume bilingüe y lo exterioriza en el desempeño donde se adhiere a que su comunicación con personas Sordas es en la lengua de señas, como un reconocimiento de lo más básico de la dignidad humana y es el derecho a una lengua que le sea propia. Logra identificar en la lengua de señas los niveles de la competencia comunicativa con la que cuentan las y los estudiantes Sordos, al ingreso y permanencia, por un lado, para propiciar el desarrollo de la lengua en la escuela y, por otro lado, para desplegar en esta un tópico o tema de clase en situaciones que permitan 
formas comunicativas que posicionen la lengua, que trasciendan los eventos cotidianos de los y las escolares. Es decir, la o el docente es autónomo en la toma de decisiones en el método comunicativo usado en estudiantes Sordos y Sordas al cerrar la puerta del aula. Sin embargo, cuando está posicionado como sujeto histórico comunicativamente en favor de la lengua de señas será bilingüe y legitimará el uso en todas sus posibilidades de la LSCh, y con complementariedad cuando sea necesario de otros métodos comunicativos, para la educación de la y el estudiante Sordo en el aula de clase.

En conjunto con docentes y codocentes, la directiva como sujeto situado en la institución armoniza los lineamientos didácticos, curriculares y comunicativos situados, con los ordenados por el Ministerio de Educación del país, en ausencia de normativa y políticas lingüísticas que posicionen la LSCh como la lengua de educación de las personas Sordas. El anterior conocimiento le permite ejercer resistencia hacia procesos de cerrar la escuela especial y enviar a estudiantes Sordos y Sordas a escuelas regulares en la fase inicial de la adquisición de la LSCh y que no cuenten con nativos hablantes o modelos lingüísticos de esta e intérpretes.

Asimismo, las formas cómo se posiciona en la institución la codocente ante su labor en el aula, en historicidad propia, con el antecedente que estudió en la misma institución y recibió terapia para hablar en formatos de la lengua oral, parte de reconocer que estas formas comunicativas le sirvieron poco para un conocimiento integral de los temas pedagógicos y de aprendizaje. Situación que la codocente se identifique con estudiantes Sordos y Sordas como un igual realizando acciones de demanda y reivindicación de la LSCh en la enseñanza. A la vez que, como codocente debe asumir un rol asignado por el docente en el aula que le permite un trabajo conjunto para desarrollar la LSCh al dar pautas de su vivencia y posibilidades de construir cultura Sorda, Sordedad, en el aula.

\section{Conclusiones}

Caracterizar la mirada sobre la lengua de señas chilena presente en la directiva, el docente y la codocente como sujetos históricos situados comunicativamente en la escuela especial, en la selección de métodos comunicativos para la educación del estudiantado Sordo, permite en el respeto otorgar un estatus lingüístico a la LSCh y a la cultura Sorda para abordar pedagógicamente los conocimientos básicos.

Por lo anterior, es importante retomar como un sujeto histórico situado comunicativamente, y en relación con las formas de uso de la LSCh que se dan en el aula, la figura de codocente Sordo en Chile sin tener en cuenta que cuente o no con una formación en educación superior. La experiencia de la práctica docente la adquiere en el aula, donde se encuentra en formación de manera simultánea en su trabajo, donde es modelo lingüístico y cultural. Este es el primer eje a ser abordado en el aula para articular el trabajo de manera colaborativa. En este sentido, debe ser la o el codocente quien apoye el uso y desarrollo de la LSCH y el o la docente la pedagogía, en diadas simétricas de colaboración y retribución de un quehacer conjunto.

El codocente, en algunas situaciones se encuentra en una doble vía laboral, contando o no con conocimientos en estrategias pedagógicas. Situación laboral doble que deben enfrentar al ejecutar, en algunos momentos, funciones docentes por necesidad o solicitud de la institución. Al tiempo que deben sobrellevar en esta función docente su propio antecedente comunicativo como sujeto histórico situado, al haber sido estudiante de la institución, y para el momento actual compartir el espacio laboral con otros docentes oyentes, en muchos casos sin alcanzar todavía el nivel de reconocimiento como colegas. En donde algunos de estos docentes oyentes fueron quienes le formaron en la enseñanza básica y con los que vivenció roces comunicativos, y que para entonces mostraron en la práctica docente un posicionamiento comunicativo en la oralidad con desconocimiento o negación de la LSCh al usar solo la lengua oral y ejercer castigos si usaba la lengua de señas.

Este panorama muestra todos los aspectos de lo que implica, en este caso para la escuela especial, la transición al uso de la LSCh en reconocimiento de un Otro diverso y la convergencia de diferentes comprensiones y niveles de uso o no la misma. 
El ingreso laboral a la escuela especial, como parte de la nómina de docentes con formación profesional y codocentes Sordos y Sordas, permite develar la omisión de la comunicación que se instauró por años en una invisibilidad de la persona Sorda, siendo retomado su ingreso como puente para vivenciar o corregir hechos y pautas que fomenten la Sordedad, como sujetos visuales, con una claridad Otra de forma innata para brindar aportes en pro del desarrollo de una pedagogía Sorda.

Al compartir directivas y docentes el espacio de clases con colegas Sordos y Sordas, en jerarquía de jefe de un curso o codocente, indudablemente, aporta a los oyentes una visibilidad Sorda en la escuela especial que viene de la anterior y se sigue con la actual dirección. Esta visibilidad Sorda permitió acciones para cuestionar y cambiar el timbre sonoro por un timbre de luz móvil o baliza. Se reconoce con esta luz giratoria la forma de comunicación visual para los manejos de los tiempos académicos y evacuación en un posible hecho de emergencia, aunque parece solo una acción aislada es toda una apuesta por la comunicación con respeto a la diversidad Sorda.

El considerar sujetos históricos situados comunicativamente es guiar acciones apuntadas a la Sordedad en proceso de identidad Sorda, que, si bien la directiva y docentes oyentes no pueden otorgar, sí pueden direccionar y contribuir de manera colaborativa para que los y las estudiantes reconozcan en edades tempranas las posibilidades que ofrece la asociación de personas Sordas como un pilar de la comunidad Sorda de desarrollo y apoyo comunicativo en todos los grupos etarios.

Para finalizar, es importante referirse a la cultura Sorda de codocentes y estudiantes en relación con la cultura oyente en la que se encuentra inserto el docente bilingüe oyente, que es minoría en la institución y se posiciona como sujeto histórico situado comunicativamente en la escuela especial, para generar situaciones académicas y poder avanzar en posicionar el camino pertinente al desarrollo de la LSCh en el aula ante los métodos comunicativos utilizados tradicionalmente en la educación de personas Sordas.

Se concluye que el docente y la codocente, con apoyo de la directiva, tienen un posicionamiento comunicativo ético para el uso de la LSCh que ha permitido al estudiantado Sordo en la escuela especial investigada acceder a todas las áreas del conocimiento en el contexto escolar. Aunque se enfrentan a la diversidad de niveles de dominio de la LSCh en el aula de clase y la correspondiente diversidad en el desarrollo de la comprensión y expresión del lenguaje lo que limita su desempeño para lo que se está enseñando y les impone roles adicionales que buscan compensar esas diversidades entre la escuela, la familia y otros contextos.

\section{ReFERENCiAS}

Acosta, L., Calvo, T., Maya, D. y Sanabria, E. (2004). Diccionario Español-Lengua de Señas Mexicana. DIELSEME. México: Dirección de Educación Especial, SEP.

Adamo, D., Acuña, X. y Cabrera I. (2009). Diccionario bilingüe lengua de señas chilenalespañol. Santiago de Chile: Universidad Metropolitana de Ciencias de la Educación y la Unidad de Educación Especial del Ministerio de Educación.

Aguirre, D (2005). Reflexiones acerca de la competencia comunicativa profesional. Educación Médica Superior, 19(3), $1-1$.

Anzola, M. (1996). Gigantes del Alma. Mérida: Ediciones del Postgrado de Lectura.

Banco Mundial. (2013). Informe anual 2013. Washington, DC: Centro Internacional de Arreglo de Diferencias Relativas a Inversiones - CIADI.

Berelson, B. (1952). Content analysis in communications research. Michigan: Free Press.

Bellés, R. (1995). Presentación: Modelos de atención educativa a los Sordos. Infancia y Aprendizaje, 18(69-70), 5-17.

Carvajal, Tascón y Vásquez. (2012). Construyendo caminos, Sordos y oyentes pensando en la U. Santiago de Cali: Universidad del Valle.

Godoy, P., Meza, M. y Salazar, A. (2004). Antecedentes históricos, presente y futuro de la educación especial en Chile. Santiago de Chile: Ministerio de Educación. 
Gina Morales-Acosta. Percepciones sobre la lengua de señas chilena en la educación de estudiantes ...

Hadjikakou, K., Christodoulou, D., Hadjidemetri, E., Konidari, M., y Nicolaou, N. (2009). The experiences of Cypriot hearing adults with deaf parents in family, school, and society. Journal of deaf studies and deaf education, 14(4), 486-502.

Ladd, P. (2011). Comprendiendo la cultura Sorda: en busca de la Sordedad. Concepción, Chile: Consejo Nacional de la Cultura y las Artes.

Mantas, R. (2014). La lengua de signos como elemento inclusor en el aula de Educación Infantil. Jaén, España: Universidad de Jaén.

Marchesi, Á., Alonso, P., Valmaseda, M. y Paniagua, G. (1995). Desarrollo del lenguaje y del juego simbólico en niños Sordos profundos. Madrid: Ministerio de Educación y Ciencia.

Massone, M. I. y Johnson, R. E. (1993). Diccionario Bilingüe Lengua de Señas Argentina-Español-Inglés. Buenos Aires: Sopena Argentina.

Massone, M., Buscaglia, V. y Cvejanov, S. (2012). Estudios Multidisciplinarios sobre las Comunidades Sordas. Buenos Aires: Universidad Nacional de Cuyo - Editorial de la Facultad de Educación Elemental y Especial Argentina.

Ministerio de Planificación. (2010). Ley 20422 que establece normas sobre igualdad de oportunidades e inclusión social de personas con discapacidad. Chile: Congreso Nacional de Chile. Recuperado de https://www.senadis.gob.cl/p ag/195/1432/ley_n20422

ONU. (2006). Convención sobre los Derechos de las Personas con Discapacidad. Recuperado de https://www.un.org/s panish/disabilities/default.asp?id $=497$

Oviedo, A. (2001). Apuntespara una gramática de la Lengua de Señas Colombiana. Colombia: Ministerio de Educación Nacional, Instituto Nacional para Sordos.

Pinto, V. (2012). Língua Brasileira de Sinais: curso de Pedagogia. Itajaí: Universidade do Vale do Itajaí.

Ramírez, A. y Masutti, M. (2009). A Educação de surdos em uma perspectiva bilíngüe: uma experiência de elaboração de softwares e suas implicações pedagógicas. Santa Catarina: Ed. da UFSC.

Salinas, A., Sánchez, J., Purcell, O. y Mendoza, C. (2009). Factores que inciden en el desarrollo y sustentabilidad de prácticas innovadoras de integración curricular de tecnologias de la información (TICs) en la sala de clases en profesores de Enseñanza Media. Santiago de Chile: Universidad de Chile. Disponible en https://bit.ly/2VWA BsE

Servicio Nacional de Discapacidad [Senadis]. (2006). Discapacidad en Chile: Pasos Hacia un Modelo Integral del Funcionamiento Humano. Gobierno de Chile.

Servicio Nacional de Discapacidad [Senadis]. (2015), Segundo estudio Nacional de Discapacidad en Chile, Gobierno de Chile.

Skliar, C. (2003). La Educación de los Sordos. Disponible en https://cultura-Sorda.org/biblioteca/articulos/educacio $\mathrm{n} / \# 2003$

Unesco. (1998). Declaración Universal de los Derechos Lingüisticos. Barcelona: Institut d'Edicions de la Diputació de Barcelona.

Unesco. (2015). Educación para Todos 2000- 2015: Logros y desafios. Francia: Ediciones Unesco.

Zemelman, H. (1998). Sujeto: existencia y potencia. México: Anthropos.

Zemelman, H. (2010). Aspectos básicos en la propuesta de la conciencia histórica (o del presentepotencial). México: Ipecal.

Zondek, D., Zepeda, S., González, M. y Recabarren, H. (2006). Discapacidad en Chile: Pasos hacia un modelo integral del funcionamiento humano. Santiago de Cali: Fondo Nacional de la Discapacidad (FONADIS).

\section{Notas}

[1] El presente trabajo se encuentra adscrito al Programa de Doctorado de Ciencias de la Educación Mención Intercultural de la Universidad Santiago de Chile (2016). 
[2] Como punto de inicio se considera importante, resaltar la escritura de la palabra Sordo y Sorda con $S$ mayúscula, que devela la enunciación y posicionamiento comunicativo en la reivindicación de la lengua de señas y el respeto a la cultura Sorda que guía este estudio y por ende al lector.

[3] Al momento de la investigación, en 2016, se reconocía que Chile solo contaba con dos colegios que posibilitaban a estudiantes Sordos y Sordas continuar estudios de secundaria en una escuela especial de para personas Sordas, es decir cursar los niveles de prebásica, básica y secundaria con pares y pedagogías para la comunidad Sorda.

\section{BY-NC-ND}

